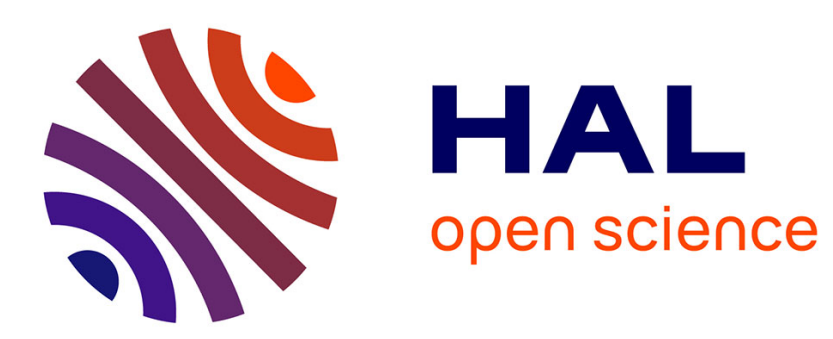

\title{
Non-equilibrium Scaling of the Turbulent-Nonturbulent Interface Speed in Planar Jets
}

\author{
Gioacchino Cafiero, J. Christos Vassilicos
}

\section{To cite this version:}

Gioacchino Cafiero, J. Christos Vassilicos. Non-equilibrium Scaling of the Turbulent-Nonturbulent Interface Speed in Planar Jets. Physical Review Letters, 2020, 125 (17), 10.1103/PhysRevLett.125.174501 . hal-03048851

\section{HAL Id: hal-03048851 \\ https://hal.science/hal-03048851}

Submitted on 15 Oct 2021

HAL is a multi-disciplinary open access archive for the deposit and dissemination of scientific research documents, whether they are published or not. The documents may come from teaching and research institutions in France or abroad, or from public or private research centers.
L'archive ouverte pluridisciplinaire HAL, est destinée au dépôt et à la diffusion de documents scientifiques de niveau recherche, publiés ou non, émanant des établissements d'enseignement et de recherche français ou étrangers, des laboratoires publics ou privés. 


\title{
Non-equilibrium scalings of the turbulent/non-turbulent interface speed in planar jets
}

\author{
Gioacchino Cafiero ${ }^{1}$ and J. Christos Vassilicos ${ }^{2}$ \\ ${ }^{1}$ Centre for Aerodynamics and Environmental Flow, \\ Department of Mechanical Engineering Sciences, \\ University of Surrey, Guildford, UK \\ ${ }^{2}$ Univ. Lille, CNRS, ONERA, Arts et Metiers Institute of Technology, \\ Centrale Lille, UMR 9014 - LMFL - Laboratoire de Mécanique \\ des fluides de Lille - Kampé de Feriet, F-59000 Lille, France
}

\begin{abstract}
The length-scale which, combined with the fluid's kinematic viscosity $\nu$, defines the local speed of the turbulent/non-turbulent interface (TNTI) has been postulated to be the smallest (Kolmogorov) length-scale $\eta$ of the turbulence (Corrsin \& Kistler 1955). This is indeed the case when the turbulence dissipation rate obeys the Kolmogorov equilibrium cascade scaling, but in the presence of the non-equilibrium turbulence dissipation scaling the local TNTI speed scales as $\nu / \lambda$, instead of $\nu / \eta$, where $\lambda$ is the Taylor length. We derive this theoretically and confirm it experimentally in the range of distances between 20 and 50 nozzle widths of a turbulent planar jet.
\end{abstract}


Interfaces are essential paradigmatic phenomena in non-equilibrium statistical physics which appear in a wide range of physical and physico-chemical contexts. The turbulent/nonturbulent interface (TNTI) is one important example of interfaces which encapsulates one of the greatest challenges in statistical physics and turbulence research: how to make sense of turbulence dynamics in the absence of homogeneity and isotropy. The TNTI is a sharp and convoluted fluctuating layer between potential non-turbulent flow on one side and vortical turbulent flow on the other. It exists at the edge of a very wide range of turbulent flows including various types of turbulent boundary layers, jets, plumes, wakes and mixing layers, and is of central relevance to mixing of mass and momentum in many environmental, geophysical and industrial contexts including combustion and cloud physics $([1,2])$.

The TNTI's motion relative to the fluid is intimately linked to entrainment. In fact, the rate at which fluid without vorticity is entrained into the turbulent core of the flow and acquires vorticity depends on the local speed of the interface relative to the fluid and on the interface's area per unit volume $[1,3]$. These two TNTI properties, local speed and area per unit volume (or length per unit area in a planar cut through the flow), are therefore key to understanding and modeling entrainment, mass and momentum fluxes, which are pivotal and pervasive fluid mechanical processes.

Corrsin \& Kistler [4] advanced the idea that the local speed of the TNTI scales with the characteristic velocity of the smallest turbulence eddies, i.e. the Kolmogorov velocity $\nu / \eta$ where $\nu$ is the fluid's kinematic viscosity and $\eta$ is the Kolmogorov lengthscale. More than 50 years later, supporting evidence was found for this hypothesis in laboratory experiments and Direct Numerical Simulations of turbulent flows where the Taylor length Reynolds number $R e_{\lambda}$ (a measure of the ratio of inertial to viscous forces) is below $100([5,6])$. Sreenivasan et al. [3] obtained evidence showing that the TNTI has fractal properties and a fractal dimension equal to $7 / 3$, thus suggesting the possibility of an asymptotically infinite TNTI area per unit volume as the Reynolds number tends to infinity. In this limit the local speed of the TNTI tends to 0 if it scales with the Kolmogorov velocity. As a result, the mass flux across the interface is finite and in fact sizeable even if the local speed is very small, and the $7 / 3$ fractal dimension reflects the scalings of the Kolmogorov velocity in order for the mass flux to remain finite.

A few years ago, Zhou \& Vassilicos [7] showed that the scalings of the local TNTI speed and the turbulence dissipation are related in the case of a self-similar turbulent axisymmetric 
wake in a way that is more general than envisaged in [4]. On account of the self-similarity, the wake width growth and the velocity deficit decay are determined by mass, momentum and turbulent kinetic energy balances and the turbulence dissipation rate is an important contributor to the latter balance [8, 9]. Different turbulence dissipation scalings lead to different wake width growth rates, and as pointed out in [7], this in turn means different local TNTI speed scalings. If the turbulence dissipation scaling is the one implied by the Kolmogorov equilibrium cascade theory, then the TNTI speed scales with the Kolmogorov velocity (as suggested in [4]) provided that the TNTI's fractal dimension is the one found in [3]; but if the turbulence dissipation obeys the non-equilibrium scaling discovered in various turbulent flows over the past ten years [8-14], then the local speed of the TNTI scales with the Taylor velocity $\nu / \lambda$ (where $\lambda$ is the Taylor length-scale) irrespective of the the TNTI's fractal dimension. The potential relevance of the Taylor length-scale to the TNTI's inner structure has also been pointed out in [15-17] but without reference to non-equilibrium turbulence cascade and dissipation.

Evidence for the non-equilibrium dissipation scaling and resulting mean flow profile scalings has been found in self-similar axisymmetric turbulent wakes $[8,9,11]$ and self-similar turbulent planar jets [14] for values of $R e_{\lambda} \equiv \sqrt{K_{0}} \lambda / \nu\left(K_{0}\right.$ is the turbulent kinetic energy on the centreline at $y=z=0$ ), larger than 100. How does the local TNTI speed scale in the region of a planar jet where self-similarity coexists with the non-equilibrium scaling? Can the prediction made for axisymmetric turbulent wakes by [7] also be made for turbulent planar jets, namely that the local TNTI speed scales with the Taylor velocity in this region, and can such a prediction be verified experimentally? These are the main questions we answer in this letter.

Figure 1a is a schematic of the main features of our experimental planar jet set-up where $h=15 \mathrm{~mm}, r=2 h, s / h=31$ and the jet inlet velocity $U_{J}$ is such that the global Reynolds number $R e \equiv U_{J} h / \nu$ is 20,000. More details can be found in [14] who used the exact same facility at the same $R e$. The jet width $\delta(x)$ at streamwise position $x$ is based on the self-similar profile of the mean streamise velocity $\langle u\rangle(x, y)=u_{0}(x) f(y / \delta(x))$ where the brackets indicate a time-average. This profile as well as a few other profiles, such as the turbulent dissipation rate's, are self-similar for $x \geq 18 h$, and statistics are homogeneous in the $z$-direction [14]. The non-equilibrium dissipation scaling which is present in turbulent planar jets was found to hold in the present jet in the range $20 h \leq x \leq 50 h$ where $R e_{\lambda}$ was 


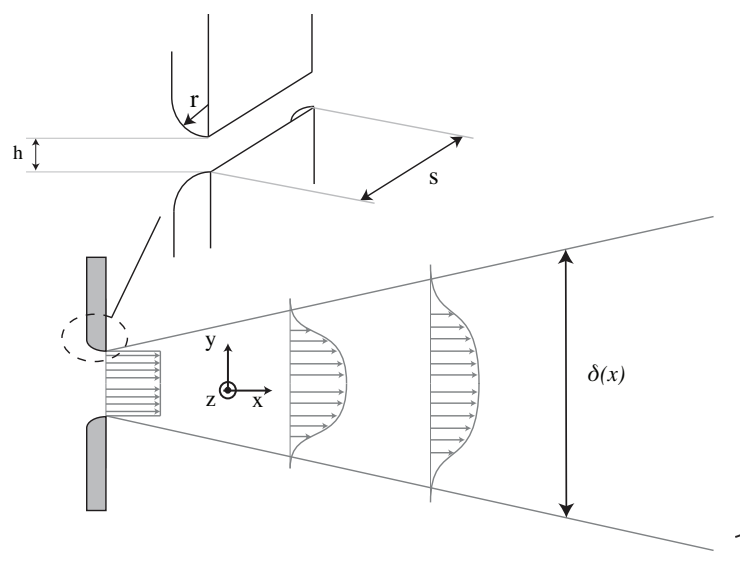

(a)

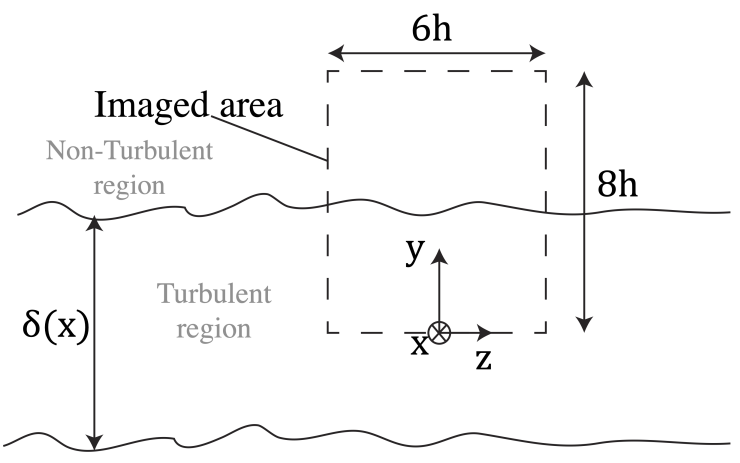

(b)

FIG. 1: a) Schematic representation of the jet flow, with indication of the reference frame; b) cross section of the jet flow at a generic streamwise distance $x$, with indication of the imaged area $0 \leq y \leq 8 h,-3 h \leq y \leq 3 h$. The two wiggly lines at the top and bottom represent the intersection of the TNTI with the $y-z$ plane at position $x$.

found to increase from 250 to 430 [14]. The region $20 h \leq x \leq 50 h$ is therefore appropriate for our experiment as it combines self-similarity, large enough values of $R e_{\lambda}$ and the nonequilibrium scaling $\varepsilon_{0} \sim U_{J} h K_{0} / \delta$ where $\varepsilon_{0}(x)$ is the turbulence dissipation rate on the centreline $y=z=0$.

The wiggly lower and upper lines in figure $1 \mathrm{~b}$ represent the intersection of the TNTI with the $y-z$ plane at a streamwise distance $x$ from inlet. The turbulent region lies between these two lines and the area imaged by our Particle Image Velocimetry (PIV) (which we describe below) is shown too.

The following relation holds for both wakes and jets [7]:

$$
\frac{d}{d x}\left\langle\int_{\mathcal{A}_{t}(x)} u d y d z\right\rangle=\mathcal{L} \bar{v}_{n}
$$

where, in the case of a planar jet (see figure $1 \mathrm{~b}), \mathcal{A}_{t}(x)$ is the instantaneous area in the $y-z$ plane at $x$ between the upper and the lower TNTI line intersections of the TNTI in a region $-Z \leq z \leq Z$ of that plane (for $Z / H$ large enough); $\mathcal{L}$ is the time-averaged length of the TNTI's intersection with this plane in this same region; and $\bar{v}_{n} \equiv\left\langle\int_{\partial_{\mathcal{A}_{t}}(x)} v_{n} d l\right\rangle / \mathcal{L}$ where $v_{n}$ is the local (in space and time) propagation speed of the TNTI relative to the fluid and the line-integration is over the line-boundary of $\mathcal{A}_{t}(x)$ in the region $-Z \leq z \leq Z$ of the $y-z$ plane at $x$. It is also worth explicitly mentioning that eq. (1) holds for any definition of an 
iso-line representing the TNTI.

The underlying concept in eq. (1) is that the mean entrainment at the TNTI is directly related to the streamwise variation of the mass flux. The use of this equation to determine the characteristic TNTI speed $\bar{v}_{n}$ requires knowledge of $\mathcal{L}$, and we know from [3] that the interfacial line between turbulent and non turbulent regions is fractal. We therefore write $\mathcal{L} \sim 2 Z\left(\eta_{I} / \delta\right)^{1-D}$ in terms of the fractal dimension $1 \leq D<2$ and the smallest length-scale $\eta_{I}$ on the interface. At this point, like in [7] for wakes, we follow Corrsin [4] and assume $\eta_{I} \sim \nu / \bar{v}_{n}$ without, however, specifying the scaling of $\bar{v}_{n}$. Using self-similarity, the left-hand side of eq. (1) scales as $\frac{d}{d x}\left(u_{0} \delta Z\right)$ and with our expression for $\mathcal{L}$, eq. (1) implies

$$
\bar{v}_{n} \sim\left(\frac{\delta}{\nu}\right)^{(1-D) / D}\left(\frac{d}{d x}\left(u_{0} \delta\right)\right)^{1 / D} .
$$

The mass, momentum and turbulent kinetic energy balance analysis of [14] for a selfsimilar planar jet showed that $u_{0} \sim\left(x-x_{0}\right)^{-a / 2}$ and $\delta \sim\left(x-x_{0}\right)^{a}$ where $a=(m+1) /(2 m+1)$ in terms of the turbulence dissipation exponent $m$ in $\varepsilon_{0} \sim\left(U_{J} h / \sqrt{K_{0}} \delta\right)^{m}\left(K_{0}^{3 / 2} / \delta\right)$. The classical dissipation scaling (which is, in particular, the scaling implied by the Kolmogorov equilibrium theory) corresponds to $m=0$ and the non-equilibrium dissipation scaling corresponds to $m=1$ [8-14]. Note that the same analysis leads to a different relation between the exponent $a$ of the wake width and the dissipation exponent $m$ for a self-similar axisymmetric wake [8]. Even so, the following conclusion, which was obtained in [7] for the axisymmetric wake, is now also reached for the planar jet:

(i) for $m=0, \bar{v}_{n} \sim \nu / \eta$ where $\eta \equiv\left(\nu^{3} / \varepsilon_{0}\right)^{1 / 4}$ is the Kolmogorov length-scale provided that $D=4 / 3(=7 / 3-1$, with $7 / 3$ being the dimension of the TNTI surface found in [3]);

(ii) for $m=1, \bar{v}_{n} \sim \nu / \lambda$ where $\lambda^{2} \equiv 10 \nu K_{0} / \varepsilon_{0}$ irrespective of the value of $D$.

In a region of a planar jet such as $20 h \leq x \leq 50 h$ where self-similarity coexists with the non-equilibrium dissipation scaling (i.e. $m=1$ ), our analysis therefore predicts that the average TNTI speed $\bar{v}_{n}$ does not scale with the Kolmogorov velocity $v_{\eta} \equiv \nu / \eta$ but scales, instead, with the Taylor velocity $v_{\lambda} \equiv \nu / \lambda$. We now proceed to verify this prediction experimentally. To the authors' knowledge, this represents the first investigation of the TNTI propagation speed scaling in a turbulent flow with a wide range of $R e_{\lambda}$ values all much higher than 100 (for a list of previous investigations on the topic, see [1]).

The interface propagation speed can be measured by tracking in time an iso-contour of the vorticity magnitude [16]. This, however, poses severe challenges, as it requires sufficiently 
high spatial resolution to correctly sample the interface and at the same time high temporal resolution in order to track in time the propagation of the interface. Another approach, requires the full vorticity vector alongside its spatial derivatives [6], which from an experimental point of view can be extremely challenging due to the effect of the measurement noise. Our approach is based, like our theory, on eq. (1) and is aimed at the measurement of $\bar{v}_{n}$. As such, it allows to relax the temporal resolution constraint and does not require spatial derivatives of vorticity.

We perform stereoscopic PIV (stereo-PIV) at four different streamwise locations to determine the kinematic and dynamic properties of the TNTI. A dual cavity/double pulsed Nd:YAG laser $(200 \mathrm{~mJ} /$ pulse, $15 \mathrm{~Hz}$ frequency, time separation between two pulses set to 90 $\mu s$ ) is used to produce a laser sheet that illuminates cross sections of the planar jet flow in the $y-z$ plane at four different streamwise locations, namely $x / h=20,30,40,50$. The two cameras (equipped with 100mm Macro lenses and Scheimpflug mounts) are located on either sides of the laser, imaging an area extending for $8 h \times 6 h$ in the $y$ - $z$ plane, starting from $y=0$ (see figure 1 ).

Equation (1) requires the calculation of the streamwise derivative of the term $\int_{\mathcal{A}_{\mathcal{T}}} u d y d z$. We perform this derivative by also acquiring stereo-PIV images at two $y-z$ planes displaced by $\pm 0.3 h$ in the $x$ direction from each measurement plane. This results in 4500 images for each of the four measurement planes, which ensures convergence of the relevant statistics.

The cross-correlation is operated using an iterative procedure with a final interrogation window size of $32 \times 32$ pixels with $75 \%$ overlap. Both raw images and velocity fields are interpolated using spline functions [18]. A Blackman filtering is applied to tune the spatial resolution [19]. The resulting vector pitch is $5 \eta$ at $x / h=20$ and reduces to $2.5 \eta$ at $x / h=50$.

Our PIV does not have sufficient resolution to calculate turbulence dissipation but as we only need the turbulence dissipation rate $\varepsilon_{0}$ on the centreline, we use the Hot Wire Anemometry (HWA) measurements carried out in [14]. The turbulence dissipation rate $\varepsilon_{0}$ was obtained from its isotropic surrogate $15 \nu \overline{\left(\partial u^{\prime} / \partial x\right)^{2}}\left(u^{\prime} \equiv u-\langle u\rangle\right.$ is the fluctuating streamwise velocity). Direct Numerical Simulations of a turbulent planar jet ([20]) have shown that the difference between $\varepsilon_{0}$ and its isotropic surrogate is negligible, particularly on the centreline.

The interface detection from PIV data is often performed by looking at threshold values of the vorticity magnitude or the magnitude of the spanwise vorticity component $\omega_{z}$. However, 
the present stereo-PIV data do not provide access to the spanwise vorticity component. Following the approach reported in [21], we look at instantaneous turbulent kinetic energy

$$
\tilde{k}=\frac{1}{9 U_{J}^{2}} \sum_{m, n=-1}^{1}\left(u_{m, n}-\langle u\rangle\right)^{2}+\left(v_{m, n}\right)^{2}+\left(w_{m, n}\right)^{2}
$$

which is a normalised (by $\left.U_{J}^{2}\right)$ average over the 9 points of a $3 x 3$ window $(m=-1,0,1$ and $n=-1,0,1)$ and where the time-average $\langle u\rangle$ is calculated at the centre of this $3 \times 3$ window. The detection of the interface in terms of $\tilde{k}$ is carried out by trying incremental threshold values $k_{t h r}$. For each of these values, the resulting contour image is discretized in binary levels 0 or 1 depending on the local value of $\tilde{k}$ being smaller or greater than $k_{t h r}$. This is repeated for each point of all instantaneous velocity fields providing us 1500 instantaneous turbulent kinetic energy fields for each $x / h=20,30,40,50$.

A contour algorithm based on this binary representation is then used to generate contour lines demarcating between regions with level 0 and regions with level 1 . These two regions can be separated by more than one continuous line, as patches of one level are often embedded within patches of the other level as illustrated in figure 2. We pick as candidate for the TNTI the longest among all the continuous lines on the image.

Watanabe et al. [22] demonstrated that iso-lines based on turbulent kinetic energy thresholds are not faithful representations of the TNTI. They are smoother than vorticity magnitude iso-lines and less sharply packed together in space for different threshold values. They are therefore not suitable for local measurements of $v_{n}$, but can nevertheless be useful for measurements of $\bar{v}_{n}$ on the basis of equation (1), which is valid for any choice of iso-line, particularly in the presence of non-equilibrium turbulence dissipation when $D$ does not influence the scaling of $\bar{v}_{n}$. There is in fact no other alternative at the present high Reynolds numbers where the necessary resolution to accurately capture velocity and vorticity derivatives cannot be achieved. The next step is to chose the value of $k_{t h r}$ which might best represent the average position of the TNTI.

Chauhan et al. [21] chose values of $k_{t h r}$ leading to a normal distribution of the intermittency function. As the fractal nature of the TNTI is important in our theoretical framework we opt to chose that value of $k_{t h r}$ which returns the best-defined fractal dimension $D$. We therefore apply a box-counting algorithm to the longest continuous line for a given $k_{t h r}$ and calculate the average number $N(r)$ of squares of size $r$ needed to cover this curve. This average is calculated over all 1500 instantaneous realisations at the given $x / h$. 


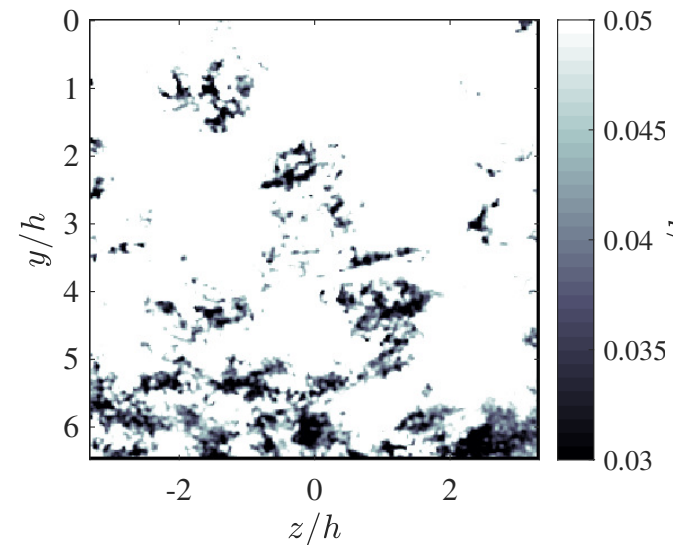

(a)

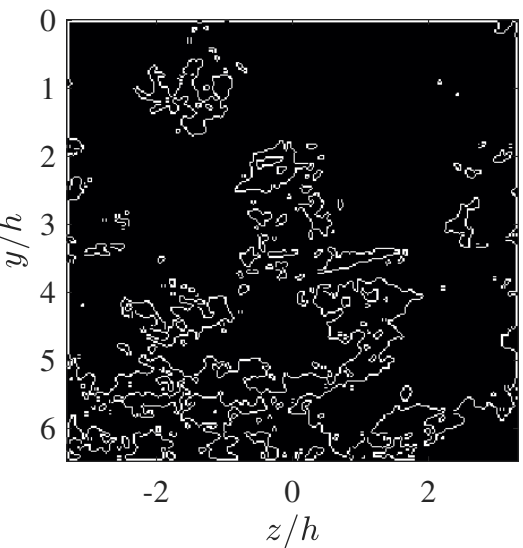

(b)

FIG. 2: a) Contour representation of an instantaneous realization of the flow color coded according to $\tilde{k}$ values. b) Edge detection using a value of $k_{t h r}=0.04$. Data are measured at

$$
x / h=40 .
$$

The fractal dimension $D$ exists and is well-defined if $N \sim r^{-D}$ over a significant range of scales $r$. We therefore plot $-\frac{\mathrm{d} \ln (N)}{\mathrm{d} \ln (r)}$ versus $\ln (r / h)$ at all $x / h=20,30,40,50$ for a wide range of $k_{t h r}$ as in figure 3a. This figure shows that, at $x / h=50$, the iso-line with the best-defined constant $D=-\frac{\mathrm{d} l n(N)}{\mathrm{d} \ln (r)}$ over a significant range of scales is the one corresponding to $k_{t h r}=0.04$. Similar plots at $x / h=20,30,40$ show that the iso-lines with the best-defined $D$ are the ones corresponding to $k_{t h r}=0.04$ for $x / h=40,50$ and $k_{t h r}=0.035$ for $x / h$ $=20,30$. This finalises our choices of $k_{t h r}$.

In figure $3 \mathrm{~b}$ we plot $-\frac{\mathrm{d} \ln (N)}{\mathrm{d} \ln (r)}$ versus $\ln (r / h)$ for our chosen iso-lines at each $x / h=$ $20,30,40,50$. The fractal dimension turns out to be $D \approx 1.2$ over the approximate range $\lambda \leq r \leq 0.2 \delta$ for all $x / h=20,30,40,50$. It should not be surprising that $D<4 / 3$ because, as shown in [22], turbulent kinetic energy iso-lines are not as irregular as vorticity magnitude iso-lines and their fractal dimension can therefore be expected to be smaller.

Using the iso-lines that we selected to represent the TNTI on the basis of their length as continuous lines and of their fractal dimension, we calculate $\mathcal{L}$ and the integral on the left side of eq. (1), and we use eq. (1) to obtain the average TNTI speed $\bar{v}_{n}$ at positions $x / h$ $=20,30,40,50$. The results are plotted in figure 4: they show that $\bar{v}_{n}$ scales with the Taylor velocity $v_{\lambda}$ and not with the Kolmogorov velocity $v_{\eta}$.

We can also use $D=1.2$ to indirectly check the Corrsin scaling $\eta_{I}=\nu / \bar{v}_{n}$ through 


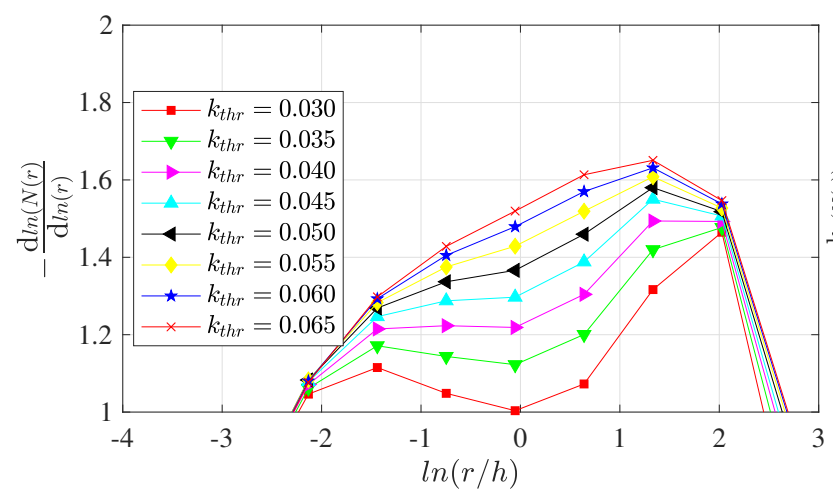

(a)

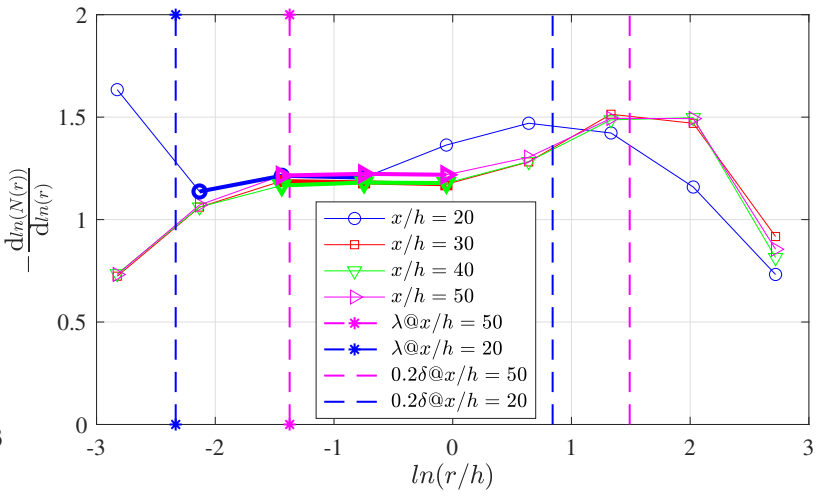

(b)

FIG. 3: a) Plot of $-\frac{\mathrm{d} \ln (N)}{\mathrm{d} \ln (r)}$ versus $\ln (r / h)$ where $N(r)$ is the time-averaged number of squares of size $r$ needed to cover the $\tilde{k}=k_{t h r}$ iso-line at $x / h=50$; data are plotted for different values of the threshold $k_{t h r}$. b) Same plot but at $k_{t h r}=0.035$ for $x / h=20,30$ and

$k_{t h r}=0.04$ for $x / h=40,50$. The vertical dashed lines are representative of the Taylor microscale $\lambda$ and of $0.2 \delta$ (in terms of the jet width $\delta$ ) at $x / h=20$ and $x / h=50$.

the relation $\mathcal{L} \sim 2 Z\left(\eta_{I} / \delta\right)^{1-D}$. We therefore calculate the ratio of $\mathcal{L}$ to $2 Z\left(\eta_{I} / \delta\right)^{1-D}$ with $\eta_{I}=\nu / \bar{v}_{n}$ where $Z=3 h$, given the size of our PIV imaged area (see figure 1 ), and where $\delta(x)$ is taken from the HWA measurements of [14] for the exact same flow at the exact same positions $x / h=20,30,40,50$. This length ratio is plotted in figure 5 and whilst it is close to constant, it exhibits a small rise from $x / h=20$ to $x / h=50$. Increasing the range over which one might consider the dimension $D$ to be well-defined, from $\lambda \leq r \leq 0.2 \delta$ to $\lambda \leq r \leq 0.4 \delta$, leads to $D \approx 1.25$ rather than $D \approx 1.2$. With this value of $D$, the ratio of $\mathcal{L}$ to $2 Z\left(\eta_{I} / \delta\right)^{1-D}$ becomes much closer to a constant (to within $1.5 \%$ of mean value without upward or downward trend) as shown in figure 5, thereby supporting Corrsin scaling of the smallest length scale on the interface by slightly increasing the fractal range of scales and, as a result, very slightly increasing the fractal dimension $D$ without changing $\bar{v}_{n}$ (which is still given by figure 4).

\section{CONCLUSIONS}

In a relatively high Reynolds number region of a turbulent planar jet characterised by the non-equilibrium turbulence dissipation scaling, the average local TNTI speed $\bar{v}_{n}$ scales 


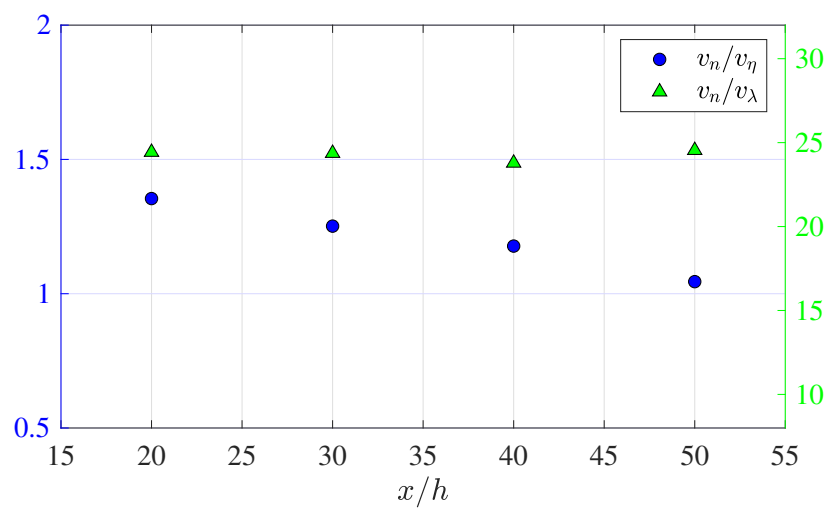

FIG. 4: Ratio of the characteristic TNTI speed $\bar{v}_{n}$ normalised by the Kolmogorov $v_{\eta}$ (full circles and left vertical axis) and by the Taylor velocity $v_{\lambda}$ (full triangles and right vertical axis)

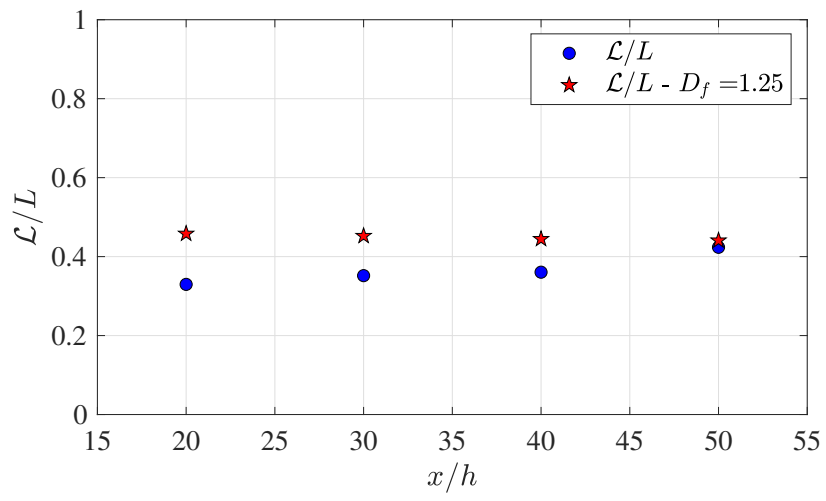

FIG. 5: Ratio $\mathcal{L} / L\left(\eta_{I}\right)$, where $L\left(\eta_{I}\right)=6 h\left(\eta_{I} / \delta\right)^{1-D}$, as a function of streamwise distance

$$
x / h, \text { for } D=1.2 \text { and } D=1.25 \text {. }
$$

with the Taylor velocity $v_{\lambda}=\nu / \lambda$ rather than the Kolmogorov velocity $v_{\eta}=\nu / \eta$. We have reached this conclusion from basic theory, and from Stereo-PIV measurements in the range of streamwise distances between 20 and $50 \mathrm{~h}$ where the non equilibrium turbulence dissipation scaling has previously been shown to hold in the exact same turbulent planar jet flow $[14]$.

The present investigation allows for the measurement of the average local TNTI speed on the basis of an averaged approach, which does not rely on high-resolution local propagation velocity measurements at the interface. This has enabled our investigation to reach Reynolds numbers higher than in previous TNTI studies and high enough for the non-equilibrium turbulence dissipation rate scaling to be present within the flow (generally clearly present 
for $\left.R e_{\lambda}>100\right)$.

The Taylor length $\lambda$ is an intermediate length which, like $\eta$, depends on viscosity but, unlike $\eta$, also depends on the turbulent kinetic energy of the turbulence. Most of this energy is in the largest turbulence eddies and potentially in large-scale coherent structures if we are not too far from inlet. In $[12,23]$ it was postulated that the non-equilibrium turbulence dissipation scaling owes its presence to coherent structures. Coherent structures can also be responsible for engulfment [1] and it may be that $\bar{v}_{n}$ scales with $\nu / \lambda$ when engulfment is an important entrainment mechanism and that $\bar{v}_{n}$ scales with $\nu / \eta$ when the dominating entrainment mechanism is nibbling [1].

\section{ACKNOWLEDGMENTS}

We were supported by the ERC Advanced Grant 320560 awarded to JCV. JCV is supported by European Union Horizon 2020 Research and Innovation Programme under the Marie Sklodowska-Curie Actions, Grant Agreement No. 675675, and Chair of Excellence CoPreFlo funded by I-SITE-ULNE/MEL/Region Hauts de France. GC acknowledges Prof. Tommaso Astarita for providing the code to perform the PIV processing.

[1] C. B. da Silva, J. C. Hunt, I. Eames, and J. Westerweel, Annual Review of Fluid Mechanics 46, 567 (2014).

[2] J. P. Mellado, Annual Review of Fluid Mechanics 49, 145 (2017).

[3] K. R. Sreenivasan, R. R. Prasad, C. Meneveau, and R. Ramshankar, Pure and Applied Geophysics PAGEOPH 131, 43 (1989).

[4] Corrsin, S. and Kistler, A.L., NACA report 1244, 1033 (1955).

[5] M. Holzner, A. Liberzon, N. Nikitin, B. Lüthi, W. Kinzelbach, and A. Tsinober, Journal of Fluid Mechanics 598, 465 (2008).

[6] M. Holzner and B. Lüthi, Physical Review Letters 106 (2011).

[7] Y. Zhou and J. C. Vassilicos, Journal of Fluid Mechanics 821, 440 (2017).

[8] T. Dairay, M. Obligado, and J. C. Vassilicos, Journal of Fluid Mechanics 781, 166 (2015).

[9] I. P. Castro, Journal of Fluid Mechanics 788, 1 (2015). 
[10] J. C. Vassilicos, Annual Review of Fluid Mechanics 47, 95 (2015).

[11] M. Obligado, T. Dairay, and J. C. Vassilicos, Physical Review Fluids 1 (2016).

[12] S. Goto and J. C. Vassilicos, Physical Review E 94 (2016).

[13] J. Nedić, S. Tavoularis, and I. Marusic, Physical Review Fluids 2 (2017).

[14] G. Cafiero and J. C. Vassilicos, Proceedings of the Royal Society A: Mathematical, Physical and Engineering Sciences 475 (2019), 1803.10488.

[15] D. K. Bisset, J. C. R. Hunt, and M. M. Rogers, Journal of Fluid Mechanics 451, 383 (2002).

[16] J. Westerweel, C. Fukushima, J. M. Pedersen, and J. C. Hunt, Physical Review Letters 95 (2005).

[17] J. Westerweel, C. Fukushima, J. M. Pedersen, and J. C. Hunt, Journal of Fluid Mechanics 631, 199 (2009).

[18] T. Astarita, Experiments in Fluids 40, 977 (2006).

[19] T. Astarita, Experiments in Fluids 43, 859 (2007).

[20] S. A. Stanley, S. Sarkar, and J. P. Mellado, Journal of Fluid Mechanics 450, 377 (2002).

[21] K. Chauhan, J. Philip, C. M. De Silva, N. Hutchins, and I. Marusic, Journal of Fluid Mechanics 742, 119 (2014).

[22] T. Watanabe, X. Zhang, and K. Nagata, Physics of Fluids 30 (2018).

[23] F. Alves Portela, G. Papadakis, and J. C. Vassilicos, Physical Review Fluids 3 (2018). 